CERN-TH.7531/94

hep-ph/9412297

\title{
Supersymmetric Unification
}

\author{
SAVAS Dimopoulos [2 \\ Theory Group, CERN \\ 1211 Geneva 23, Switzerland
}

\begin{abstract}
The measured value of the weak mixing angle is, at present, the only precise experimental indication for physics beyond the Standard Model. It points in the direction of Unified Theories with Supersymmetric particles at accessible energies. We recall the ideas that led to the construction of these theories in 1981.
\end{abstract}

\footnotetext{
${ }^{1}$ Talk presented at the International Conference on the History of Original Ideas and Basic Discoveries in Particle Physics held at Ettore Majorana Centre for Scientific Culture, Erice, Sicily, July 29-Aug.4 1994.

${ }^{2}$ On leave of absence from the Physics Department, Stanford University, Stanford Ca. 94305, USA. Work supported in part by NSF Grant NSF-PHY-9219345
} 


\section{Why Supersymmetric Unification?}

It is a pleasure to recall the ideas that led to the first Supersymmetric Unified Theory and its low energy manifestation, the Supersymmetric $S U(3) \times S U(2) \times$ $U(1)$ model. This theory synthesizes two marvelous ideas, Unification [1] and Supersymmetry [2, 3]. The synthesis is catalyzed by the hierarchy problem [4] which suggests that Supersymmetry occurs at accessible energies [5]. Since time is short and we are explicitly asked to talk about our own contributions I will not cover these important topics.

A look at the the program of this Conference reveals that most other topics covered are textbook subjects, such as Renormalization of the Standard Model [6] and Asymptotic Freedom [7], that are at the foundation of our field. So it is natural to ask why Supersymmetric Unification is included in such a distinguished company of well-established subjects? I am not certain. Perhaps the main reason at present is a quantitative prediction, dating from 1981, that has been verified by high precision data (see figure); that is a correlation between $\alpha_{s}\left(M_{Z}\right)$ and $\sin ^{2}\left(\theta_{W}\right)$ which has been confirmed by experiment at the $1 \%$ level [8]. In fact this is the only significant quantitative success of any extension of the Standard Model, and is the strongest experimental hint that we have for physics beyond the Standard Model .

It is amusing that this prediction of the weak mixing angle, at the time it was made, disagreed with experiment [9] (see figure). Experiments since then, especially the recent LEP results, have convincingly changed the experimental world average in favor of the prediction. The success of this prediction depends crucially on having both Unification and low energy Supersymmetry in the same theory; either Unification or Supersymmetry alone are insufficient. So, although we have not seen any superparticles yet, we have evidence for Superunification !

We will discuss the developments in chronological order, beginning with the state of our field before 1981.

\section{Before 1981.}

A crucial turning point in our field occurred in the Spring of 1978. The SLAC experiment on parity violation in neutral currents convinced many theorists that the Standard Model of Glashow, Weinberg and Salam was correct and that it was a good time to start focusing on the next layer of questions: to explain some of the puzzling features of the Standard Model. The first question that theorists turned to was the "hierarchy problem" 田: attempting to understand why the Higgs mass is so much smaller than the Planck mass or the Unification Scale. The Higgs does not carry any symmetry that ensures its lightness; indeed, in 
Figure: The correlation in the values of $\sin ^{2}\left(\theta_{W}\right)$ and $\alpha_{s}\left(M_{Z}\right)$ predicted in SUSYGUTs and ordinary GUTs. The bare Superstring prediction is the point on the far right. The present 1994 data are contrasted with the 1981 data. The bands are the uncertainties in the theoretical predictions of GUTs and SUSY-GUTs. The numbers in the bands indicate the Unification Scale. The uncertainties in the theoretical predictions for superstrings are not known.

the absence of miraculous cancellations, the Higgs mass would be driven to the Planck or unification scale; it would not be available at low energies to do its intended job of giving mass to the weak gauge bosons.

Susskind and Weinberg [10] proposed the very appealing idea of Technicolor, as an alternative to the Higgs, for giving mass to the weak gauge bosons. In early '79 Technicolor was enlarged into "Extended Technicolor" [11] to allow the quarks and leptons to get their masses. By the summer of 1980 it became clear that these theories suffered from generic problems of flavor violations 12 that could perhaps be cured only by complicating the theory immensely and losing any hope of calculability. I, perhaps prematurely, felt that this was too high a price to pay and decided to look at other alternative approaches to the Hierarchy problem. 
That is when we turned to Supersymmetry [2, 3]. It was generally realized that Supersymmetry could help the hierarchy problem [0]. The reason is that the Higgs, a scalar, would form a degenerate pair with a fermion, called the Higgsino. Since the Higgsino could be protected by a chiral symmetry from becoming superheavy, so could its degenerate scalar partner, the Higgs. Of course Supersymmetry does much more than to just relate the Higgs to the Higgsino. It assigns a degenerate scalar "superpartner" to each and every known quark and lepton, as well as a fermionic degenerate superpartner to each gauge boson. Since no such particles had been seen it was clear that Supersymmetry had to be a broken symmetry. Nevertheless, Supersymmetry would still help the hierarchy problem as long as its breaking occurs near the weak scale, the superpartners are at accessible energies ! This line of reasoning led us to begin our attempt to find a Supersymmetric version of the Standard Model with Supersymmetry broken at the weak scale. Together with Stuart Raby and Leonard Susskind we started learning about Supersymmetry and tried to find out if such theories had already been constructed. We quickly discovered that no Supersymmetric versions of the Standard Model existed at that time. There were early attempts by Fayet [13] that were plagued by the following problems:

- They were not Supersymmetric extensions of the Standard $S U(3) \times S U(2) \times$ $U(1)$ model. The gauge group was $S U(3) \times S U(2) \times U(1) \times U(1)^{\prime}$. Without the extra $U(1)^{\prime}$ neutral current interactions, the theory had phenomenological problems: it spontaneously violated electric charge and color conservation.

- The extra neutral current $U(1)^{\prime}$ was anomalous. To get a consistent theory one had to cancel the anomalies by introducing extra light particles, distinct from the ordinary quarks and leptons. These again led to color and electric charge breaking at the weak scale: the photon had a mass $\sim M_{W} \cdot{ }^{3}$

The root of these problems was that in these theories Supersymmetry was broken spontaneously at the tree level. In 1979 a very important paper by Ferrara, Girardello and Palumbo [14 showed that in such theories, under very general conditions, color and charged scalars would get negative masses squared, leading to breaking of electric charge and color. This essentially stopped efforts to build realistic Supersymmetric theories.

During the fall of 1980 these difficulties often caused us to wonder whether we were not on the wrong track and Supersymmetry was doomed to fail in the real world, at least as a low energy phenomenon. Almost nothing that we tried

\footnotetext{
${ }^{3}$ There were other problems such as a continuous R-symmetry which forced the gluino to be massless.
} 
worked. We could not cancel the anomalies of the extra $U(1)^{\prime}$ without giving a mass $\sim M_{W}$ to the photon and gluon. The extra $U(1)^{\prime}$ made it impossible to unify', which is important for addressing the hierarchy problem and for predicting $\sin ^{2}\left(\theta_{W}\right)$. We also had no clue as to what to do with the continuous R-symmetry that implied massless gluinos. Little of what we learned during these early exercises has survived. The benefit was that we learned bits of the mathematical formalism which told us, loud and clear, that all known particles have superpartners and how these couple . However, given all the problems, it was unclear that anything that we learned would survive.

Since the origin of these problems was that Supersymmetry was broken spontaneously, it seemed clear to us that we should look for alternate mechanisms to break Supersymmetry. At first, we attempted to break Supersymmetry dynamically with a new strong force, very similar to Technicolor, which we called Supercolor. We were not alone in these efforts. Witten [5] as well as Dine, Fischler and Srednicki [5] were pursuing similar ideas for precisely the same reasons. They wrote two very important papers entitled "Dynamical breaking of Supersymmetry "(Witten) and "Supersymmetric Technicolor" (Dine, Fischler and Srednicki). Their preprints appeared in April of ' 81 at the same time as our "Supercolor" paper [5]. An essential objective of these works was to point out that low energy Supersymmetry helps the hierarchy problem gue that a new strong force analogous to QCD or Technicolor may induce the breaking of Supersymmetry and explain the smallness of the electroweak scale. Dine, Fischler and Srednicki, as well as Raby and myself, also attempted to build explicit models incorporating these ideas, but without much success. I do not have time to discuss these "Supercolor" or "Supersymmetric Technicolor" theories. They had problems; one of them was that they were baroque. By January of 1981 we were very discouraged. Although Stuart Raby and I had begun writing the Supercolor paper [5], we already did not believe in it. It seemed too much to believe that Nature would make simultaneous use of Supersymmetry and Technicolor to solve the hierarchy problem.

\section{1.}

\footnotetext{
${ }^{4}$ This was not a problem for the early attempts [13]; Unification was not one of their objectives since they had not associated Supersymmetry with the hierarchy problem.

${ }^{5}$ Hypercharge anomalies dictated an even number of Higgs doublets. Later, $\sin ^{2}\left(\theta_{W}\right)$ forced the number of doublets to be 2 [16].

${ }^{6}$ We were, for sure, not alone in being aware of this. Several theorists, in addition to those in Reference [5], knew it and did not publish it. The problem was to implement the idea in a consistent theory, incorporating Supersymmetry at low energies.
} 


\subsection{Distancing Ordinary Particles from the Origin of Su- persymmetry Breaking.}

In January of 1981 the prospects for a realistic Supersymmetric model were not bright. Models with spontaneusly broken Supersymmetry had grave phenomenological problems. Dynamical Supersymmetry breaking models were at best baroque. The prevailing view was that a realistic Supersymmetric model would not be found until the problem of Supersymmetry Breaking was solved. It was further believed that the experimental consequences of Supersymmetric theories would strongly depend on the details of the mechanism of Supersymmetry breaking. After all, it was this mechanism that caused the phenomenological disasters of the early attempts.

The key that took us out of this dead end grew out of our protracted frustration with the above problems, and our desire to do physics with the idea of superunification. These - quite suddenly - led us to switch problems, adopt a more phenomenological approach and simply assume that the dynamics that breaks Supersymmetry is external to and commutes with the ordinary $S U(3) \times S U(2) \times U(1)$ sector; specifically, we postulated that:

1. The only particles carrying $S U(3) \times S U(2) \times U(1)$ quantum numbers are the ordinary ones and their Superpartners that reside at the weak scale. Extra particles with exotic $S U(3) \times S U(2) \times U(1)$ quantum numbers are unnecessary.

2. The ordinary particles and their superpartners do not carry any extra new gauge interactions at low energies. This is essential for SU(5) unification.

3. The sole effect of the Supersymmetry breaking mechanism is to lift the masses of the supersymmetric partners of all ordinary particles to the weak scale.

These hypotheses helped us sidestep the obstacles that stood in the way of Unifying and doing physics in the ordinary $S U(3) \times S U(2) \times U(1)$ sector, the domain of experimental physics ! The question of the origin of Supersymmetry Breaking had been circumvented; a good thing, since this question continues to remain open. These hypotheses started bearing fruits immediately. 


\subsection{Raby and Wilczek: "Supersymmetry and the Scale of Unification."}

In this paper [15] we computed the Unification Mass when you have a minimal Supersymmetric particle content. We found that, because the superpartners of the gauge bosons slow down the evolution of the couplings, the unification mass increased to about $10^{18} \mathrm{GeV}$. This was interesting for two reasons:

- This value is close to the Planck mass, perhaps suggesting eventual unity with gravity?.

- There was a distinct experimental difference with ordinary $S U(5)$ : the proton lifetime was unobservably long.

The latter appeared to be an easily disprovable prediction. In fact by that time three different experimental groups had reported preliminary proton decay "candidate events": the Kolar gold field, Homestake mine and the Witwatersrand experiments. We knew that S.Miyake, of the Kolar Gold Field experiment, and possibly representatives of the other experiments were going to talk about their events in the upcoming "Second Workshop on Grand Unification" where I was also going to present our theoretical results. So, I was a bit nervous but did not hesitate for a moment to present them. I was, and still am, very proud of this paper. A simple and well motivated ingredient, virtual superparticles, made a huge difference to a quantity that was being measured at that time, the proton lifetime. Perhaps this is the first test that SUSY-GUTs have passed. In this paper, although we pointed out that the value of $\sin ^{2}\left(\theta_{W}\right)$ would change due to the Higgs sparticles, we did not present the new value. After satisfying ourselves that it would not be grossly modified, we focused on the change in the unification mass, which at that time was more important for experiment.

The next big step was to construct a realistic supersymmetric theory.

\footnotetext{
${ }^{7}$ This connection got weaker as more accurate calculations [8, 16, 18] reduced the value to $\sim 2 \times 10^{16} \mathrm{GeV}$.
} 


\subsection{Georgi: "Supersymmetric GUTs."}

These two papers [16] titled "Softly Broken Supersymmetry and SU(5)" and " Supersymmetric GUTs" accomplished three objectives:

1. Supersymmetric Unification (SUSY-GUTs): Construction of a Unified supersymmetric theory of strong and electroweak forces. Our gauge group was $\mathrm{SU}(5)$. This was not really much harder than building a nonunified theory. Unification was also essential for the prediction of $\sin ^{2}\left(\theta_{W}\right)$ and for some of the phenomenology, such as proton decay and gaugino masses. It was also a good framework for addressing the hierarchy problem.

2. Supersymmetry Breaking: Supersymmetry was broken softly but explicitly by mass terms for all scalar superpartners and gauginos. The origin of supersymmetry breaking was not specified. As long as it is external to and commutes with $S U(3) \times S U(2) \times U(1)$ it does not matter for experiment ${ }^{8}$. Ingenious ideas for generating these soft terms by either new gauge forces [23], or via supergravity [25] were proposed a year later.

3. Supersymmetric Standard Model (SSM): As a bonus, our theory contained the first phenomenologically viable supersymmetric extension of the standard $S U(3) \times S U(2) \times U(1)$ model $(\mathrm{SSM})$.

We constructed the model in late March and early April of 1981. We were overjoyed. We had the first realistic Supersymmetric theory, incorporating all non-gravitational phenomena and valid up to the Planck mass. We immediately started thinking about experimental consequences. We wanted to make sure that we would not miss anything important. Time pressure helped us a lot. Both Howard and I were scheduled to give two consecutive talks in the Second Workshop on Grand Unification which took place at the University of Michigan on April 24-26, 1981. Here are some of our phenomenological results that we reported in that Workshop [16]:

\footnotetext{
${ }^{8}$ See Sections 3.1 and 5.
} 
- $\sin ^{2}\left(\theta_{W}\right)$ : We presented our SUSY-GUT prediction for $\sin ^{2}\left(\theta_{W}\right)$. The magnitude we got disagreed with the central experimental value, but the errors were large. We argued that there would have to be 2 Higgs doublets for the value not to be too far off.

- Proton Decay: We reported that the Supersymmetric Unification Mass is so large [15] that proton decay is unobservably small.

- Superparticle Spectroscopy: squarks and sleptons. We postulated that all squarks and sleptons have a common universal mass $\left(\sim M_{W}\right)$ at the unification scale. This way we had a Super-GIM mechanism supressing flavor violations. The Higgses had different masses.

- Superparticle Spectroscopy: gauginos. Because we had a unified theory all gauginos had a common Majorana mass $\left(\sim M_{W}\right)$ at the unification scale.

- Family Reflection Symmetry; Stable LSP. To avoid rapid proton decay via dimension-four operators we postulated a discrete symmetry forbidding three-family couplings. This symmetry was subsequently called family reflection symmetry 20] or matter parity. We concluded:

"the lightest of the supersymmetric particles is stable. The others decay into it plus ordinary particles. One simple possibility is that it is the U(1) gauge fermion.' 1

It is gratifying that the above ingredients have survived the test of time. They form the basis of what is now called the minimal supersymmetric standard model (MSSM) T. Perhaps the most important conclusion of our paper is also the one that now seems so evident because it has, with time, been incorporated into our thinking:

\footnotetext{
${ }^{9}$ Continuous symmetries, such as Technibaryon number [10] or continuous R-symmetry [13] also lead to new stable particles. In fact, many extensions of the SM have this feature.

${ }^{10}$ The acronym MSSM is often used incorrectly to mean minimal SUSY-GUTs. Gell-Mann's terms "Superstandard model" and "Superunified theory" are much better but not widely used.
} 
"The phenomenology of the model is simple. In addition to the usual light matter fermions, gauge bosons and Higgs bosons, we predict heavy matter bosons, gauge fermions and Higgs fermions as supersymmetric partners. We can say little about their mass except that they cannot be very large relative to 1 Tev or the motivation for the model disappears." 16]

Of course, our motivation was to address the hierarchy problem; without it we could not have drawn this conclusion.

Georgi and I spoke on the last day of the conference [9]. My feeling was that our results were for the most part ignored, especially by the experimentalists who did not care about the hierarchy problem. Our conclusions were very much against the spirit of the conference. There were three things against us:

- The central value of the weak mixing angle agreed better with the predictions of ordinary (non-Supersymmetric) Grand Unified Theories, albeit with large error bars (see figure).

- Preliminary proton decay "candidate events" had been reported by three different experimental groups, the Kolar gold field, Homestake mine and the Witwatersrand experiments.

- The host institution was gearing up to launch the biggest effort on protondecay namely the IMB experiment.

The atmosphere in the conference is summarized by Marciano's April 24, 1981 concluding remarks [9]:

"The basic idea of Grand Unification is very appealing. The simplest model based on $S U(5)$ has scored an important success in predicting a value for $\sin ^{2}\left(\theta_{W}\right)$ which is in excellent agreement with recent experimental findings (after radiative corrections are included). It makes an additional dramatic prediction that the proton will decay with a lifetime in the range of $10^{30}-10^{32}$ years. If correct, such decays will be seen by the planned experiments within the coming year (or may have already been seen). An incredible discovery may be awaiting us." "凹

It is remarkable that Georgi, in such an atmosphere, did not hesitate to propose an alternative to his and Glashow's '74 theory [1] which seemed to be on the verge of being proven. But this is Howard! He would rather have fun with physics than worry about such things.

\footnotetext{
${ }^{11}$ The emphasis here is mine.
} 
Very significant encouragement came from Sheldon Glashow, Leonard Susskind and Steven Weinberg. In his April 26, 1981 conference summary talk [9] Weinberg mentioned our theory and its predictions of $\sin ^{2}\left(\theta_{W}\right)$ and $M_{G U T}$ several times. His verdict [9]:

"...the model of Dimopoulos and Georgi has many other attractive features and something like it may turn out to be right."

This was music to my ears.

In May I presented our results in two more conferences, one in Santa Barbara and the other at the Royal Society in London. Soon afterwards theoretical activity in supersymmetric unification began to pick up. In August of '81 Girardello and Grisaru wrote a very important paper [17] systematically discussing explicit soft breaking of global supersymmetry; they were the first to discuss cubic soft terms. Starting in July of ' 81 several important papers [18] with the calculation of the superunified value of $M_{G U T}$ and $\sin ^{2}\left(\theta_{W}\right)$ appeared, some improving it to two loops. Sakai's paper 18 includes an analysis of $\mathrm{SU}(5)$ breaking which is very similar to ours; it does not introduce the soft superparticle mass terms that break supersymmetry and thus does not address the phenomenology of superparticles.

The interest in GUTs and SUSY-GUTs dwindled after 1983. The rise of

superstrings, the absence of proton decay and the lack of precise data on $\sin ^{2}\left(\theta_{W}\right)$ were some of the reasons. The morale among the non-stringers was so low that the annual series of "Workshops on Grand Unification" was terminated. 1989 was the year of the "Last Workshop on Grand Unification". In the introduction to that terminal volume Paul Frampton exclaimed:

"Alas, none of the principal predictions of GUTs have been confirmed." This was written in August 1989, just as LEP was beginning to take data...

\section{Proton Decay Revisited.}

Although Georgi and I worried a lot about dimension-four baryon violating operators and we introduced the family reflection symmetry to forbid them, it did not occur to us to check the operators of dimension five ! Weinberg 19 as well as Sakai and Yanagida [19] studied these operators and concluded that they pose a severe problem for our theory. They attempted to construct models with an extra $U(1)^{\prime}$ gauge group that would forbid the dimension five operators that mediated proton decay. Raby, Wilczek and I studied these operators in October of '81 and concluded that the small Yukawa couplings of the light 
generation naturally supressed these operators [20]. The resulting proton decay rates, although not calculable from low energy physics parameters, could be experimentally observable. Furthermore they had a very unique signature that is not expected in non-supersymmetric theories: protons and neutrons decay into kaons. We were very excited that we had identified another "smoking gun" for supersymmetry. Ellis, Nanopoulos and Rudaz independently reached the same conclusions [20].

\section{Completing the Picture.}

Since time is so short I have limited myself to those aspects of superunified theories that are least model-dependent and experimentally testable or, in the case of $\sin ^{2}\left(\theta_{W}\right)$ and proton decay, perhaps already tested. Of course, the theory that we proposed is far from complete and left many important theoretical questions unanswered. I will briefly mention some of the problems and related ideas.

Doublet-triplet splitting: There is one remaining technically natural fine tuning in our theory [16]. Wilczek and I addressed this problem in June of 1981 and found two solutions now called the missing partner and the missing VEV mechanisms [21]. Attempts to implement these mechanisms in realistic theories led to very complicated constructions [22]. This continues to be an open problem.

Hidden sector: The theoretical question of how supersymmetry is broken and superparticle masses are generated in our theory attracted a lot of attention. Georgi and I had spent a couple of days thinking about this and then decided that it was not phenomenologically interesting: two different theories of supersymmetry breaking that give precisely the same soft masses to the superpartners of ordinary particles cannot be experimentally distinguished. So we abandoned it. Our philosophy was to build an effective theory that describes the $S U(5)$ part of supersymmetric world, which is accessible to experiment. It could result from many different ways of breaking Supersymmetry, as long as the postulates of Section 3.1 are satisfied.

Nevertheless, it was important to present at least an existence proof of a mechanism that generated our soft terms. An important consideration was that squarks and sleptons belonging to different generations had to have identical masses to avoid problems with rare processes [16]. In the winter/spring of ' 82 three different groups [23], Dine and Fischler, Raby and I, and Polchinski and Susskind came up with the idea of a Hidden Sector, around $10^{11} \mathrm{GeV}$, where supersymmetry breaking originates and is subsequently communicated to the 
ordinary particles via a new gauge interaction at the unification scale afterwards a series of very important papers developed a better idea for such a mechanism: Supersymmetry breaking could be communicated from the hidden sector via supergravity 25].

Radiative electroweak breaking: Hidden sector mechanisms for Supersymmetry breaking, under very special assumptions, give degenerate masses to all scalars: squarks, sleptons as well as Higgses. This is good for avoiding flavor violations [16] but poses the puzzle: what distinguishes the Higgs from the squarks and the sleptons? Why does the Higgs get a vacuum expectation value and not the squarks? [7]. Starting with Ibañez and Ross, a series of very important papers [26] developed the idea of radiative electroweak breaking which answers this question dynamically provided the top quark is sufficiently heavy, above $\sim 60 \mathrm{GeV}$.

The title of this section is misleading. The picture is still very far from complete; many fundamental questions remain unanswered. The theory we have is definitely not a theory of everything. Instead, it is a phenomenological, disprovable theory that allows us to make contact with experiment in spite of the questions that it fails to address.

\section{Prospects.}

In the last few minutes I want to take a break from history and mention some contemporary issues.

\subsection{How significant is the $\sin ^{2}\left(\theta_{W}\right)$ prediction?}

SUSY-GUTs: Since the LEP data confirmed the SUSY-GUT prediction this topic has received a lot of attention and is discussed in many papers. Excellent recent analyses are those of Ref. [\&]. We summarize the results in the table and the figure which together with their captions tell the story. The estimated uncertainties in the theoretical predictions for SUSY-GUTs and GUTs are due to: $\alpha_{s}\left(M_{Z}\right)$ and $\alpha\left(M_{Z}\right)$ error bars, sparticle thresholds, $m_{t}$ and $m_{h^{0}}$, GUT thresholds and Non-renormalizable operators at the unification scale. For the $\sin ^{2}\left(\theta_{W}\right)$ prediction they all add up to about $\pm 1 \%$ [ [ ] The experimental error is negligible, $\pm 0.2 \%$. The probability that the agreement is an accident is $\sim 2 \%$. The largest source of theoretical uncertainty is due to the $\alpha_{s}\left(M_{Z}\right)$ error bar; this

\footnotetext{
${ }^{12}$ For Raby and me the starting point was trying to build a realistic model utilizing Witten's idea of "Inverted Hierarchy" 24.

${ }^{13}$ In the original SUSY-GUT this was not an issue because the Higgs masses were assumed to be different from the universal squark and slepton masses [16].

${ }^{14} \sin ^{2}\left(\theta_{W}\right)$ is in the $\overline{\mathrm{MS}}$ scheme.
} 
Table: The experimental values for $\sin ^{2}\left(\theta_{W}\right)$ and $\alpha_{s}\left(M_{Z}\right)$ are contrasted with the predictions of three theories: Ordinary GUTs, SUSY-GUTs and bare Superstrings . Under each prediction we list the number of standard deviations that it differs from experiment. GUTs and SUSY-GUTs predict one of either $\sin ^{2}\left(\theta_{W}\right)$ or $\alpha_{s}\left(M_{Z}\right)$; the other one is an input. For Strings both $\sin ^{2}\left(\theta_{W}\right)$ and $\alpha_{s}\left(M_{Z}\right)$ are predictions. The uncertainties in the theoretical predictions for superstrings are not known.

should shrink in the future. The other uncertainties are significantly smaller. The threshold corrections are proportional to $\alpha$ s times logarithms of mass ratios. For example, the total of the low energy sparticles' contributions is summarized in the following elegant expression [8, 27]:

$$
\sin ^{2} \theta\left(M_{Z}\right)=0.2027+\frac{0.00365}{\alpha_{3}\left(M_{Z}\right)}-\frac{19 \alpha_{e m}\left(M_{Z}\right)}{60 \pi} \ln \left(\frac{T_{S U S Y}}{M_{Z}}\right)
$$

where四,

$$
T_{S U S Y}=m_{\widetilde{H}}\left(\frac{m_{\widetilde{W}}}{m_{\tilde{g}}}\right)^{28 / 19}\left[\left(\frac{m_{\tilde{l}}}{m_{\tilde{q}}}\right)^{3 / 19}\left(\frac{m_{H}}{m_{\widetilde{H}}}\right)^{3 / 19}\left(\frac{m_{\widetilde{W}}}{m_{\widetilde{H}}}\right)^{4 / 19}\right] .
$$

and $m_{\tilde{q}}, m_{\tilde{g}}, m_{\tilde{l}}, m_{\widetilde{W}}, m_{\widetilde{H}}$ and $m_{H}$ are the characteristic masses of the squarks, gluinos, sleptons, electroweak gauginos, Higgsinos and the heavy Higgs doublet, respectively. $T_{S U S Y}$ is an effective SUSY threshold.

From these equations we learn that the supersymmetric threshold corrections are typically small. The same holds for the high energy threshold corrections in minimal SUSY-GUTs [8]. Therefore the $\sin ^{2} \theta\left(M_{Z}\right)$ prediction is quite insensitive to the details of both the low and the high mass-scale physics; it takes a number of highly split multiplets to change it appreciably. For example, we know that to bring $\sin ^{2} \theta\left(M_{Z}\right)$ down by just $\sim 10 \%$ — back to the standard $\mathrm{SU}(5)$ value - we would need to lift the higgsinos and the second higgs to $\sim 10^{14} \mathrm{GeV}$.

\footnotetext{
${ }^{15}$ In eq.(2) if any mass is less than $M_{Z}$ it should be replaced by $M_{Z}$.
} 
The flip side of these arguments show that to "fix" Standard GUTs, you also need several highly split multiplets [28]. In fact you need many more, since you do not have superpartners. The figure and the table show that in Standard GUTs either $\sin ^{2}\left(\theta_{W}\right)$ or $\alpha_{s}\left(M_{Z}\right)$ are off by many standard deviations. Worse yet, the proton decays too fast. Do these problems mean that all non-supersymmetric GUTs are excluded? Of course not. By adding many unobserved split particles at random to change the running of the couplings you can accommodate just about any values of $\sin ^{2}\left(\theta_{W}\right)$ and $M_{G U T}$. So, in what sense are these quantities predicted?

I answer this with a quote from Raby and Wilczek [29]:

"Once we wander from the straight and narrow path of minimalism, infinitely many silly ways to go wrong lie open before us. In the absence of some additional idea, just adding unobserved particles at random to change the running of the couplings is almost sure to follow one of these. However there are a few ideas which do motivate definite extensions of the minimal model, and are sufficiently interesting that even their failure would be worth knowing about.'四

The '81 predictions for $\sin ^{2}\left(\theta_{W}\right)$ and $M_{G U T}$ were inevitable consequences of an idea; they could not be modified, although they came at a time when they were least expected and, for sure, unwanted.

Peaceful coexistence with Superstrings: The predictions that we quote in the table for superstrings assume the minimal supersymmetric particle content up to the string scale $M_{s}$ of about $4 \times 10^{17} \mathrm{GeV}$, and do not include any potentially large string-induced corrections $\$$. These corrections are model dependent; in the absense of a model, it is not possible to estimate their magnitude. It is clear that the corrections would have to be quite large to make up for the large discrepancies with experiment. It is possible that a model will be found where the corrections are large and can be tuned to accommodate the data. Such a "fix" seems no better than accomodating ordinary SU(5) with large corrections caused by random unobserved multiplets. Perhaps a more appealing solution will be found. Such a solution should answer the question posed by Barbieri et al. 30]:

\footnotetext{
${ }^{16}$ Emphasis mine

${ }^{17}$ The bare string value of $\alpha_{s}\left(M_{Z}\right)=0.2$ gives a proton mass of approximately $20 \mathrm{GeV}$. This follows directly from $M_{s} / M_{G U T} \simeq 20$.
} 
"why should these corrections maintain the relations between the couplings characteristic of the Grand Unified symmetry, if such a symmetry is not actually realised?"

One possibility is that at $M_{s}$ the string theory breaks to a SUSY-GUT [30, 31] ; this is a promising new direction which may combine some of the virtues of both SUSY-GUTs and strings. A challenge to such attempts would be to explain the ratio of the SUSY-GUT scale to the string scale.

\subsection{Life before LHC}

Where are the Sparticles? The short answer is still:

“...We can say little about their mass except that they cannot be very large relative to 1 Tev or the motivation for the model disappears." 116

Upper limits can be obtained which are functions of the amount of fine tuning that you allow in the theory [32], but this is clearly a matter of taste. For any fixed amount of fine tuning a large top Yukawa coupling pushes down many sparticle masses; so these upper limits are now known to be near their minima. For example, if you only allow $10 \%$ fine tunings then the two lightest neutralinos and chargino would be accessible at LEP 200.

Obviously, sparticle masses are proportional to the SUSY breaking scale. In contrast, the lightest Higgs mass has logarithmic sensitivity to the SUSY scale since its mass is proportional to the weak VEV - which of course is fixed, after the requisite fine tuning. As a result, even if the sparticles are at $\sim 10 \mathrm{TeV}$ the upper limit to its mass is only $\sim 160 \mathrm{GeV}$ [33, 27] ; it drops to $\sim 120 \mathrm{GeV}$ if the sparticles are below a $\mathrm{TeV} \cdot 1 \mathrm{~B}$

If we are really lucky then, before LHC is built, we may see sparticles at LEP 200 or proton decay into kaons at SuperKamiokande or Icarus. What if we are not? There are still some other possible consequences of SUSY-GUTs. These have to do with rare processes.

Hall-Kostelecky-Raby effects: The postulated universality of the masses of sparticles belonging to different generations [16] suppresses rare processes. Universality means that the sparticle mass matrix is "isotropic" in flavor space; only the quark masses spoil this isotropy by virtue of their differing eigenvalues and $V_{K M}$. As a result, just like in the SM, all flavor violating quantities involve the usual left handed angles and phase of $V_{K M}$ and are under control. In GUTS there are more physical angles and phases by virtue of transitions caused by the extra gauge particles. In ordinary GUTs these do not matter at low energies;

\footnotetext{
${ }^{18}$ In contrast to SUSY-GUTs, in the SM vacuum stability gives a lower bound to the Higgs mass of $\approx 135 \mathrm{GeV}$.
} 
they decouple like $M_{G U T}^{-1}$. Not so in SUSY-GUTs ! [34]. The sparticle masses are distorted by these extra angles and phases and propagate new flavor violations down to low energies, especially if there are large Yukawa couplings such as the top quark's 35. These can lead to interesting flavour or CP violating effects, even in minimal SUSY-GUTs, such as $\mu \rightarrow e$ transitions [35] and electric dipole moments for the neutron $\left(d_{n}\right)$ and electron $\left(d_{e}\right)$ [36][9. These already put interesting bounds on sparticle parameters. Experimentalists are encouraged to look for these effects in the near future.

These effects are expected to be even bigger, perhaps too big, in theories that explain flavour [37 suggesting that sparticles are too heavy to be accessible at LHC. Is it possible to have light sparticles and these effects suppressed? Yes. The soft sparticle masses could be truly "soft" and disappear at high energies"?; then they cannot sense the extra flavor physics occuring at $M_{G U T}$. One would only give up the usual lore where supersymmetry is fed from the hidden sector via supergravity ${ }^{21}$. This would change none of the experimental consequences of the '81 softly broken SUSY-GUTs.

\section{Acknowledgments}

I would like to thank: R. Barbieri, M.Carena, G.Giudice, N.Polonsky and C. Wagner for very valuable conversations; Ann Georgi and her family for having my family as houseguests during two very exciting weeks in March and April of '81; Professor Harvey Newman and his family, Professor Zichichi, Professor Ypsilantis and the friendly people of Erice for organizing a marvelous conference and for their hospitality.

\footnotetext{
${ }^{19}$ These effects, just like $\sin ^{2}\left(\theta_{W}\right)$, are only logaritmically sensitive to the GUT scale ! Unlike $\sin ^{2}\left(\theta_{W}\right)$, they depend on powers of the yet unknown sparticle masses.

${ }^{20}$ SUSY breaking could be communicated by particles much lighter than $M_{G U T}$ to the visible sector.

${ }^{21}$ In this case the soft masses are definitely "hard": they persist up to the Planck scale and they can sense the extra flavor physics.
} 


\section{References}

[1] H. Georgi and S. Glashow, Phys. Rev. Lett. 327 (1974) 438;

J. Pati and A. Salam, Phys. Rev. D 8 (1973) 1240;

H. Georgi, H. Quinn and S. Weinberg, Phys. Rev. Lett. 33 (1974) 451.

[2] Yu. A. Gol'fand and E.P. Likhtman, JETP Lett. 13 (1971) 323;

D.V. Volkov and V.P. Akulov, Phys. Lett. B 46 (1973) 109;

J. Wess and B. Zumino, Nucl. Phys. B 70 (1974) 39.

[3] D.Z. Freedman, P. van Nieuwenhuizen, and S. Ferrara, Phys. Rev. B 13 (1976) 3214;

S. Deser and B. Zumino, Phys. Lett. B 62 (1976) 335.

[4] K. Wilson, as mentioned in L. Susskind, Phys. Rev. D 20 (1979) 2619;

E. Gildener, Phys. Rev. D 14 (1976) 1667;

E. Gildener and S. Weinberg, Phys. Rev. D 15 (1976) 3333.

[5] L. Maiani, Proceedings of the Summer School of Gif-Sur-Yvette (Paris 1980);

M. Veltman, Acta Phys. Polon. B 12 (1981) 437;

S. Dimopoulos and S. Raby, Nucl. Phys. B 192 (1981) 353;

E. Witten, Nucl. Phys. B 188 (1981) 513;

M. Dine, W. Fischler, and M. Srednicki, Nucl. Phys. B 189 (1981) 575;

ibid., B 202 (1982) 238.

[6] See G.'tHooft, these proceedings.

[7] See D.Gross, these proceedings.

[8] Excellent recent analyses are: P. Langacker and N. Polonsky, Phys. Rev. D 47 (1993) 4028;

ibid., D 49 (1994) 1454;

L.J. Hall and U. Sarid, Phys. Rev. Lett. 70 (1993) 2673.

[9] The Second Workshop on Grand Unification, University of Michigan, Ann Arbor, April 24-26, 1981, eds. J.Leveille, L.Sulak, D.Unger; Birkhauser, 1981.

[10] S. Weinberg, Phys. Rev. D 13 (1976) 974; D 19 (1979) 1277;

L. Susskind, Phys. Rev. D 20 (1979) 2619.

[11] S. Dimopoulos and L. Susskind, Nucl. Phys. B 155 (1979) 237 ;

E. Eichten and K. Lane, Phys. Lett. B 90 (1980) 125. 
[12] S. Dimopoulos, S. Raby and P. Sikivie, Nucl. Phys. B 219, (1982) 479;

S. Dimopoulos and J. Ellis, Nucl. Phys. B 182 (1981) 505.

[13] P. Fayet, Phys. Lett. B 69 (1977) 489;

B 84 (1979) 416.

[14] S. Ferrara, L. Girardello, and F. Palumbo, Phys. Rev. D 20 (1979) 403.

[15] S. Dimopoulos, S. Raby, and F. Wilczek, Phys. Rev. D 24 (1981) 1681.

[16] S. Dimopoulos and H. Georgi, "Supersymmetric GUTs", p 285, Second Workshop on Grand Unification, University of Michigan, Ann Arbor, April 24-26, 1981, eds. J.Leveille, L.Sulak, D.Unger; Birkhauser, 1981;

S. Dimopoulos and H. Georgi, Nucl. Phys. B 193 (1981) 150.

[17] L. Girardello and M.T. Grisaru, Nucl. Phys. B 194 (1982) 65.

[18] N. Sakai, Zeit.Phys. C 11 (1981) 153;

L. Ibañez and G.G. Ross, Phys. Lett. B 105 (1981) 439;

M. B. Einhorn and D. R. T. Jones, Nucl. Phys. B 196 (1982) 475;

W. J. Marciano and G. Senjanovic, Phys. Rev. D 25 (1982) 3092.

[19] S. Weinberg, Phys. Rev. D 26 (1982) 287;

N. Sakai and T. Yanagida, Nucl. Phys. B 197 (1982) 533.

[20] S. Dimopoulos, S. Raby, and F. Wilczek, Phys. Lett. B 112 (1982) 133;

J. Ellis, D.V. Nanopoulos, and S. Rudaz, Nucl. Phys. B 202 (1982) 43.

[21] S. Dimopoulos and F. Wilczek, Santa Barbara preprint, July 1981; Proceedings Erice Summer School, Ed. A. Zichichi (1981).

[22] B. Grinstein, Nucl. Phys. B 206 (1982) 387;

R.N. Cahn, I. Hinchliffe, and L. Hall, Phys. Lett. B 109 (1982) 426;

A. Masiero, D.V. Nanopoulos, K. Tamvakis, and T. Yanagida, Phys. Lett. B 115 (1982) 380;

K.S. Babu and S.M. Barr, Phys. Rev D 48 (1993) 5354;

D 50 (1994) 3529.

[23] M. Dine, W. Fischler, Nucl. Phys. B 204 (1982) 346;

S. Dimopoulos and S. Raby, Nucl. Phys. B 219 (1982) 479;

J. Polchinski and L. Susskind, Phys. Rev. D 26 (1982) 3661.

[24] E. Witten, Phys. Lett. B 105 (1981) 267. 
[25] E. Cremmer, S. Ferrara, L. Girardello, and A. Van Proyen, Phys. Lett. B 116 (1982) 231;

A. Chamseddine, R. Arnowitt, and P. Nath, Phys. Rev. Lett. 49 (1982) 970;

R. Barbieri, S. Ferrara, and C. Savoy, Phys. Lett. B 110 (1982) 343;

L. J. Hall, J. Lykken, and S. Weinberg, Phys. Rev. D 27 (1983) 2359.

[26] L.E. Ibañez and G.G. Ross, Phys. Lett. B 110B (1982) 215;

L. Alvarez-Gaumé, M. Claudson, and M.B. Wise, Nucl. Phys. B 207 (1982) 96 ;

M. Dine and W. Fischler, Nucl. Phys. B 204 (1982) 346;

K. Inoue, A. Kakuto, H. Komatsu, and S. Takeshita, Prog. Theor. Phys. 68 (1982) 927 and 71 (1984) 413;

J. Ellis, D.V. Nanopoulos, and K. Tamvakis, Phys. Lett. B 121 (1983) 123;

L. Alvarez-Gaumé, J. Polchinski, and M. Wise, Nucl. Phys. B 221 (1983) 495;

L.E. Ibañez and C. Lopez, Phys. Lett. B 126 (1983) 54;

Nucl. Phys. B 233 (1984) 511;

C. Kounnas, A.B. Lahanas, D.V. Nanopoulos, and M. Quiros, Nucl. Phys.

B 236 (1984) 438;

L.E. Ibañez, C. Lopez, and C. Munoz, Nucl. Phys. B 256 (1985) 218;

G. Gamberini, G. Ridolfi, and F. Zwirner, Nucl. Phys. B 331 (1990) 331.

[27] M. Carena, S. Pokorski, and C.E.M. Wagner, Nucl. Phys. B 406 (1993) 59.

[28] P.H. Frampton and S.L. Glashow, Phys. Lett. B 131 (1983) 340, Erratum B 135 (1984) 515;

A. Giveon, L.J. Hall, and U. Sarid, Phys. Lett B 271 (1991) 138.

[29] S. Dimopoulos, S. Raby, and F. Wilczek, Phys. Today 44 (1991) 25-33.

[30] R. Barbieri, G. Dvali, and A. Strumia, Pisa preprint: IFUP-PTH-94-22;

[31] G. Aldazabal, A. Font, L.E. Ibañez, and A.M. Uranga, Madrid preprint: FTUAM-94-28;

S. Chadhouri, S.-W. Chung, and J.D. Lykken, Fermilab-pub-94-137-T;

G.Cleaver, OSU preprint.

[32] J. Ellis, K. Enqvist, D.V. Nanopoulos, and F.Zwirner, Mod. Phys. Lett. A 1 (1986) 57 ;

R. Barbieri and G.F. Giudice, Nucl. Phys. B 306 (1988) 63.

[33] Y. Okada, M. Yamaguchi, and T. Yanagida, Prog. Theor. Phys. Lett. 85 (1991) 1 ; 
J. Ellis, G. Ridolfi, and F. Zwirner, Phys. Lett. B 257 (1991) 83 and B 262 (1991) 477;

H.E. Haber and R. Hempfling, Phys. Rev. Lett. 66 (1991) 1815;

R.Barbieri and M. Frigeni, Phys. Lett. B 258 (1991) 395.

[34] L.J. Hall, V. A. Kostelecky, and S. Raby, Nucl. Phys. B 267 (1986) 415.

[35] R. Barbieri and L.J. Hall, LBL-36022 (1994).

[36] S.Dimopoulos and L.J.Hall, LBL-36040 (1994).

[37] H.Georgi, Phys. Lett. B 169 (1986) 231. 
This figure "fig1-1.png" is available in "png" format from: http://arxiv.org/ps/hep-ph/9412297v1 
This figure "fig1-2.png" is available in "png" format from: http://arxiv.org/ps/hep-ph/9412297v1 